\title{
DESENVOLVIMENTO VEGETATIVO, PRODUÇÃO E QUALIDADE DE FRUTOS DA TANGERINA 'FREMONT' SOBRE QUATRO PORTA-ENXERTOS ${ }^{1}$
}

\author{
ERICK ESPINOZA NÚÑEZ² FRANCISCO DE ASSIS ALVES MOURÃO FILHO ${ }^{3}$, EDUARDO SANCHES STUCHI ${ }^{4}$
}

RESUMO - Com o objetivo de avaliar o desenvolvimento vegetativo, produção e qualidade de frutos da tangerina 'Fremont' $(C$. clementina Hort. ex Tan. X C. reticulata Blanco) sobre os porta-enxertos limão 'Cravo' ( $C$. limonia Osbeck), citrumelo 'Swingle' $(P$. trifoliata Raf. x C. paradisi Macf.), tangerina 'Cleópatra' (Citrus reshni Hort. ex Tan.) e tangelo 'Orlando' (C. reticulata Blanco x C. paradisi Macf.), foi instalado um experimento em Bebedouro-SP, em 1997. Embora diferenças na produção tenham sido registradas em função dos porta-enxertos, nos anos de 2003 a 2005, a produção acumulada nas safras de 2000 a 2006 não revelou influência dos mesmos. Os valores do índice de alternância de produção e eficiência de produção não foram influenciados pelos porta-enxertos. O teor de sólidos solúveis, bem como acidez total foram superiores nos frutos das plantas enxertadas sobre citrumelo 'Swingle' e tangerina 'Cleópatra'. Os valores de volume da copa e diâmetro do tronco foram superiores nas árvores sobre tangelo 'Orlando' e tangerina 'Cleópatra'.

Termos para indexação: Citrus, propagação, vigor

\section{GROWTH, YIELD AND FRUIT QUALITY OF 'FREMONT’ MANDARIN ON FOUR ROOTSTOCKS}

\begin{abstract}
An experiment was installed in Bebedouro, SP, Brazil in 1997, with the aim of evaluate the effect of the rootstocks 'Rangpur' lime (C. limonia Osbeck), 'Swingle' citrumelo (P. trifoliata Raf. x C. paradisi Macf.), 'Cleopatra' mandarin (Citrus reshni Hort. ex Tan.) and 'Orlando' tangelo (C. reticulata Blanco $x$ C. paradisi Macf.) on plant growth, yield and fruit quality of 'Fremont' mandarin (C. clementina Hort. ex Tan. X C. reticulata Blanco). Despite that differences in yield have been registered related to the rootstocks between 2003 to 2005; cumulative yield from 2000 to 2006 seasons was not affected by the rootstock. Alternate bearing index and yield efficiency values were not influenced by the rootstocks. Fruits from trees on 'Swingle' citrumelo and 'Cleópatra' mandarin had higher values of total soluble solids concentration and total acids. Trunk diameter and canopy volume values were higher in trees on 'Orlando' tangelo and 'Cleopatra' mandarin.
\end{abstract}

Index terms: Citrus, propagation, vigour

\section{INTRODUÇÃO}

A produção de tangerinas e seus híbridos no mundo atingiu 23,3 milhões de toneladas em 2005. A China é o maior produtor, com $49 \%$ da produção mundial, em que a cultivar mais produzida é a tangerina 'Poncã'. O Brasil produziu 1,3 milhão de toneladas no mesmo período (FAO, 2006).

Apesar de o consumo per capita de frutos de tangerina ter crescido, existe um enorme potencial de mercado para os anos seguintes pela tendência de consumir alimentos saudáveis e a preocupação da indústria em desenvolver e introduzir novas cultivares que satisfaçam à expectativa dos consumidores (Boteon \& Neves, 2005). As principais cultivares e híbridos de tangerinas atualmente cultivados no Brasil, por ordem de área plantada, são a tangerina 'Poncã' (Citrus reticulata Blanco) (58\%), o tangor 'Murcott' [Citrus sinensis (L.) Osbeck X Citrus reticulata Blanco] (23\%), a tangerina 'Cravo' (Citrus reticulata Blanco) (11\%) e a tangerina 'Montenegrina' (Citrus deliciosa Tenore) (8\%) (Amaro \& Caser, 2003), sendo os maiores Estados produtores, São Paulo, Rio Grande do Sul, Paraná e Minas Gerais (IBGE, 2006).

A mancha-marrom-de-alternaria está presente em diversos países produtores de citros (Timmer et al., 1998). No Brasil, foi relatada pela primeira vez em 2001, por Goes et al. (2001), em pomares de tangerina 'Dancy' e, em 2002, estava presente em diferentes estados brasileiros (Spósito et al., 2003). O controle da mancha-marrom-de-alternaria é feito principalmente por fungicidas, método considerado difícil e oneroso devido ao grande número de aplicações que são necessárias para garantir frutos de boa qualidade. De acordo com Perez \& Timmer (2005), pode-se chegar a 12 o número de aplicações com fungicidas à base de cobre em tangor 'Murcott' no Brasil. A tangerina 'Fremont' (C. clementina Hort. ex Tan. x C. reticulata Blanco) não mostrou sintomas da doença (Reis, 2006).

Os porta-enxertos afetam mais de 20 características da árvore e dos frutos. No Brasil, o porta-enxerto mais utilizado para as tangerinas é o limão ‘Cravo' (Citrus limonia Osbeck), devido a sua tolerância ao vírus da tristeza dos citros (CTV), elevado

\footnotetext{
${ }^{1}$ (Trabalho 179-06). Recebido em : 13-11-2006. Aceito para publicação em : 16-03-2007.

${ }^{2}$ Eng $^{\circ}$ Agr $^{\circ}$, Aluno do PPG em Fitotecnia (Mestrado), Universidade de São Paulo, ESALQ, Piracicaba-SP. eenunez@esalq.usp.br

${ }^{3}$ Eng $^{\circ}$ Agr $^{\circ}$, Dr., Professor Associado. Universidade de São Paulo, ESALQ, Departamento de Produção Vegetal 9, 13418-900, Piracicaba-SP. famourao@esalq.usp.br (autor correspondente)

${ }^{4}$ Eng $^{\circ}$ Agr $^{\circ}$, Dr., Pesquisador Científico A. Embrapa Mandioca e Fruticultura Tropical, Cruz das Almas-BA, Diretor Científico. Estação Experimental de Citricultura de Bebedouro-SP. stuchi@estacaoexperimental.com.br
} 
vigor no viveiro, grande desenvolvimento das árvores enxertadas, tanto no viveiro quanto no campo, rápida entrada em produção, alto rendimento, maturação precoce, compatibilidade com todas as cultivares cítricas e principalmente sua capacidade para induzir resistência à seca (Pompeu Júnior, 2005; Salibe \& Moreira, 1984). Por outro lado, várias pesquisas têm indicado que o limão 'Cravo' não induz boa qualidade de fruto, principalmente para cultivares destinadas ao mercado de fruta fresca (Castle et al., 1993; Castle, 1995; Wutscher, 1988). O surgimento de doenças e a possível limitação do potencial produtivo são os principais problemas da utilização de um único porta-enxerto (Pompeu Júnior, 2005).

O objetivo desta pesquisa foi avaliar o desenvolvimento vegetativo, produção e qualidade de frutos da tangerina 'Fremont' sobre quatro porta-enxertos, nas condições edafo-climáticas de Bebedouro-SP.

\section{MATERIAL E MÉTODOS}

Os trabalhos experimentais foram realizados na Estação Experimental de Citricultura de Bebedouro (EECB), no município de Bebedouro-SP (latitude 20 53' 16" S, longitude 48 28' $11^{\prime}$ ' W, altitude $601 \mathrm{~m}$ ), em um solo classificado como Latossolo Vermelho, distrófico típico, textura média, A moderado, hipoférrico. O clima é do tipo Cwa (subtropical com inverno moderado e seco, verão quente e chuvoso), com temperatura máxima de $28,8^{\circ} \mathrm{C}$, média mínima $18,3{ }^{\circ} \mathrm{C}$ e temperatura média de $23,5^{\circ} \mathrm{C}$; a precipitação anual é de $1.522 \mathrm{~mm}$. As árvores foram plantadas em 30 de outubro de 1997, em densidade de 595 plantas/ha (7,0 m x 2,4 m). O pomar não foi irrigado, nem podado e foi conduzido de acordo com as práticas culturais recomendadas (Mattos Junior et al., 2005). O pomar experimental está rodeado por outros experimentos de avaliação de plantas cítricas.

A tangerina 'Fremont', um híbrido entre tangerina 'Clementina' (C. clementina Hort. ex Tan.) e tangerina 'Poncã' (C. reticulata Blanco), foi enxertada sobre os porta-enxertos: limão 'Cravo' (Citrus limonia Osbeck), citrumelo 'Swingle' $(P$. trifoliata Raf. x C. paradisi Macf.), tangelo 'Orlando' ( $C$. reticulata Blanco $x$ C. paradisi Macf.) e tangerina 'Cleópatra' (Citrus reshni Hort. ex Tan.).

$\mathrm{Na}$ data da colheita (geralmente em julho de cada ano), pesou-se a produção total de frutos de cada planta. Calculou-se a produção acumulada para todos os anos. A qualidade de cinco frutos por árvore foi determinada no Laboratório de Qualidade da EECB. A massa dos frutos foi determinada em uma balança com sensibilidade de 5,0 g. O suco foi extraído por extratora OIC modelo OTTO 1800 e calculou-se a porcentagem do suco pela relação massa do suco / massa do fruto. O teor de sólidos solúveis totais (SST) foi determinado por leitura direta no refratômetro. A acidez total (AT) foi obtida por titulação de $25 \mathrm{ml}$ de suco com hidróxido de sódio 0,3125 N. O Ratio, relação aritmética entre SST e AT, também foi calculado. O volume de copa (V) foi calculado, uma vez que foi medida a altura da planta (H), o diâmetro da copa no sentido da linha (Dl) e no sentido perpendicular à rua (Dr), em agosto de 2006, pela fórmula: $\mathrm{V}=(ð / 6)$ x H x Dl x Dr (Turrel, 1946 apud Fallahi \& Rodney, 1992). O diâmetro do tronco foi medido na cultivar copa, $10 \mathrm{~cm}$ acima do ponto de enxertia, em agosto de 2006. A eficiência da produção foi estimada dividindo-se a produção de frutos pelo volume da copa de cada porta-enxerto, em 2006. Devido à irregularidade da produção dos primeiros anos, o índice de alternância da produção (IAP) foi calculado entre os anos 2003 e 2006, usando-se a seguinte fórmula (Hoblyn et al., 1936, apud Smith et al., 2004): IAP $=1 / n-1 \times\left\{\left|\left(a_{2}-a_{1}\right)\right| /\left(a_{2}+a_{1}\right)+\left|\left(a_{3}-a_{2}\right)\right| /\right.$ $\left.\left(\mathrm{a}_{3}+\mathrm{a}_{2}\right)+\ldots+\left|\left(\mathrm{a}_{(\mathrm{n})}-\mathrm{a}_{(\mathrm{n}-1)}\right)\right| /\left(\mathrm{a}_{(\mathrm{n})}+\mathrm{a}_{(\mathrm{n}-1)}\right)\right\}$, em que $\mathrm{n}=$ número de anos e $\mathrm{a}_{1}, \mathrm{a}_{2}, \ldots, \mathrm{a}_{(\mathrm{n}-1)}, \mathrm{a}_{(\mathrm{n})}=$ produção dos anos correspondentes. $\mathrm{O}$ delineamento foi o inteiramente casualizado, com cinco repetições e uma planta por parcela. A produção por anos, produção acumulada, diâmetro do tronco, volume de copa, eficiência da copa, índice de alternância da produção e a qualidade dos frutos foram submetidos à análise de variância, e as médias foram comparadas pelo teste de Tukey $(P \leq 0,05)$.

\section{RESULTADOS}

Nos três primeiros anos de produção de frutos, não ocorreram diferenças entre as árvores sobre os quatro portaenxertos (Tabela 1). Em 2003, as plantas sobre limão 'Cravo' apresentaram produção superior às sobre os outros portaenxertos. Em 2004, as plantas sobre o limão 'Cravo' foram mais produtivas do que as sobre citrumelo 'Swingle', sem diferenciarse daquelas sobre tangelo 'Orlando' e tangerina 'Cleópatra'. Em 2005 , as plantas sobre citrumelo 'Swingle' apresentaram a maior produção de frutos quando comparadas às sobre limão 'Cravo'. Em 2006, não se registraram diferenças em função dos portaenxertos na produção de frutos. No acumulado total e bianual (2005 e 2006) da produção de frutos, não ocorreram diferenças em função dos porta-enxertos. Não foram observadas diferenças em função dos porta-enxertos para o índice de alternância da produção (Tabela 1). Os maiores valores de diâmetro do tronco e volume de copa foram registrados em plantas sobre tangelo 'Orlando', sem diferir daquelas sobre tangerina 'Cleópatra', e as menores ocorreram naquelas sobre limão 'Cravo' e citrumelo 'Swingle'. Quanto à eficiência de produção, não houve diferenças em função dos quatro porta-enxertos. Em 2005, observou-se que a massa dos frutos de árvores sobre limão 'Cravo' foi superior àquelas sobre tangerina 'Cleópatra', sem se diferenciar daquelas sobre tangelo 'Orlando' e citrumelo 'Swingle' (Tabela 2). Em relação à porcentagem de suco dos frutos, não se observaram diferenças em função dos porta-enxertos, nas safras de 2005 e 2006. Em 2005, os SST em frutos de plantas sobre citrumelo 'Swingle' foram similares aos de frutos de plantas em tangerina 'Cleópatra' e superiores aos frutos de plantas em limão 'Cravo' e tangelo 'Orlando'. Em 2006, os valores dos SST das plantas sobre citrumelo 'Swingle’ não diferiram daquelas sobre limão 'Cravo' e tangerina 'Cleópatra', mas foram superiores àquelas sobre tangelo 'Orlando'. Em 2005, os valores de AT em frutos de plantas sobre citrumelo 'Swingle' foram superiores aos demais portaenxertos, enquanto em 2006, não diferiram daquelas sobre tangerina 'Cleópatra'. Em 2005, não foram observadas diferenças no ratio dos frutos em função dos quatro porta-enxertos, enquanto em 2006, as plantas sobre limão 'Cravo' induziram ratio mais elevado (Tabela 2). 


\section{DISCUSSÃO}

$\mathrm{Na}$ produção acumulada de frutos, não ocorreram diferenças em função dos porta-enxertos, fato que concorda com várias pesquisas anteriores (Figueiredo et al., 2000; Parente et al., 1993; Pompeu Júnior et al., 2003; Souza et al., 1992). O citrumelo 'Swingle' e o limão 'Cravo' induziram menor diâmetro de tronco e volume de copa, enquanto o tangelo 'Orlando' e a tangerina 'Cleópatra' foram os porta-enxertos mais vigorosos. Stenzel et al. (2003) observaram maior desenvolvimento da tangerina 'Poncã' sobre a tangerina 'Cleópatra', fato também relatado por Figueiredo et al. (2001) para o tangor 'Murcott' e por Parente et al. (1993) para a tangerina 'Poncã'. Por outro lado, Hearn \& Hutchison (1977) observaram maior desenvolvimento das árvores das tangerinas 'Page' e 'Robinson' sobre os portaenxertos tangelo 'Orlando' e tangerina 'Cleópatra'. Amaral et al. (1995) e Pompeu Júnior et al. (2003) concluíram que as árvores de tangor 'Murcott' e laranja 'Hamlim' desenvolveram mais vigorosamente sobre as tangerinas do que as sobre limão 'Cravo'. Embora as árvores mais vigorosas tenham maior potencial produtivo, pode ocorrer sombreamento entre as mesmas, levando à diminuição na eficiência da produção, uma vez que a faixa produtiva da copa está localizada numa camada externa de um metro de profundidade e que capta $90 \%$ da radiação solar (Wheaton et al., 1978). Os dados deste experimento reforçam a idéia de que os porta-enxertos não influenciam na alternância da produção, isto é, essa característica seria afetada principalmente pela cultivar copa. Estes dados são similares aos descritos por Smith et al. (2004) em experimento de 26 anos para o tangor 'Ellendale', na região subtropical da Austrália, onde se comparou o efeito de sete porta-enxertos sobre a alternância de produção. Também Georgiou (2000) avaliou a tangerina 'Nova' sobre onze porta-enxertos, não observando diferenças em função dos porta-enxertos nessa característica. Por outro lado, Stenzel et al. (2003), avaliando a tangerina 'Poncã' no Paraná, concluíram que a alternância da produção foi menor sobre citrange C13 ou
Trifoliata. Spósito et al. (1998) recomendavam o uso de portaenxertos de vigor médio para diminuir a incidência da alternância de produção, em relação a porta-enxertos que apresentam crescimento débil ou muito vigoroso. Tendo os porta-enxertos pouco ou nenhum efeito sobre a alternância da produção, outras técnicas devem ser utilizadas para amenizar esse problema, como o raleio de frutos, o anelamento de ramos, as aplicações exógenas de reguladores vegetais e controlar a época da colheita, pois o seu atraso reduz a floração do ano seguinte (Spósito et al., 1998; Wheaton, 1997). A massa do fruto e a porcentagem do suco não foram influenciadas pelos porta-enxertos. Neste trabalho, citrumelo 'Swingle' e tangerina 'Cleópatra' conferiram melhor qualidade aos frutos das copas, especialmente no conteúdo de SST e AT. Estes resultados são apoiados por pesquisas anteriores (Wutscher \& Shull, 1972). A tangerina 'Cleópatra' tem sido o porta-enxerto mais utilizado na Flórida para as tangerinas porque confere bom tamanho de fruta, boa qualidade de suco e maior tempo de armazenamento na árvore (Castle \& Gmiter, 1998; Hearn \& Hutchison, 1997; Ouko \& Abubaker, 1988). Entretanto, o citrumelo 'Swingle' é também um excelente porta-enxerto, caso as condições de solo e umidade sejam favoráveis, como ocorreu neste experimento. Diferenças inerentes à fisiologia das árvores devido aos porta-enxertos podem ajudar a explicar as diferenças na acumulação de carboidratos entre frutos de diferentes portaenxertos. As árvores vigorosas têm maior assimilação líquida de $\mathrm{CO}_{2}\left(\mathrm{~A}_{\mathrm{CO} 2}\right)$ do que aquelas menos vigorosas. Além disso, pode haver uma diferença na partição dos fotoassimilados entre árvores sobre porta-enxertos de vigor diferente (Syvertsen \& Lloyd, 1994). A assimilação de $\mathrm{CO}_{2}$ e a partição dos fotoassimilados podem ser controladas pelas diferenças nas relações hídricas devido à distribuição radicular, eficiência de absorção da água (Castle \& Krezdorn, 1973), diâmetro das radicelas (Huang \& Eissenstat, 2000), condutividade hidráulica (Syvertsen \& Graham, 1985) e acumulação de solutos via ajuste osmótico (Barry et al., 2004).

TABELA 1 - Produção anual e acumulada de frutos, índice de alternância de produção (IAP), diâmetro de tronco, volume de copa e eficiência de produção de árvores da tangerina 'Fremont' sobre quatro porta-enxertos, em Bebedouro-SP (2000-2006).

\begin{tabular}{|c|c|c|c|c|c|c|c|c|c|c|c|c|}
\hline \multirow{2}{*}{ Porta-enxerto } & \multicolumn{7}{|c|}{$\begin{array}{c}\text { Produção (Kg/planta) } \\
\text { Ano }\end{array}$} & \multirow{2}{*}{$\begin{array}{c}\text { Produção } \\
\text { acumulada } \\
\text { 2000-06 } \\
\text { (Kg/planta) }\end{array}$} & \multirow{2}{*}{$\begin{array}{c}\text { IAP } \\
2003-06\end{array}$} & \multirow{2}{*}{$\begin{array}{c}\text { Diâmetro } \\
\text { tronco } \\
2006 \\
(\mathrm{~cm})\end{array}$} & \multirow{2}{*}{$\begin{array}{c}\text { Volume } \\
\text { copa } \\
2006 \\
\left(\mathrm{~m}^{3}\right)\end{array}$} & \multirow{2}{*}{$\begin{array}{c}\text { Eficiência } \\
\text { produção } \\
2006 \\
\left(\mathrm{Kg} / \mathrm{m}^{3}\right)\end{array}$} \\
\hline & 2000 & 2001 & 2002 & 2003 & 2004 & 2005 & 2006 & & & & & \\
\hline Limão ‘Cravo' & $14,4 \mathrm{a}$ & $3,1 \mathrm{a}$ & $24,5 \mathrm{a}$ & $27,1 \mathrm{a}$ & $21,8 \mathrm{a}$ & $12,2 \mathrm{~b}$ & $58,5 \mathrm{a}$ & $161,7 \mathrm{a}$ & $0,4 \mathrm{a}$ & $7,8 \mathrm{~b}$ & $5,4 \mathrm{~b}$ & $10,9 \mathrm{a}$ \\
\hline Citrumelo 'Swingle' & $8,9 \mathrm{a}$ & $2,1 \mathrm{a}$ & $20,1 \mathrm{a}$ & $7,0 \mathrm{~b}$ & $5,9 \mathrm{~b}$ & $26,5 \mathrm{a}$ & $53,8 \mathrm{a}$ & $124,3 \mathrm{a}$ & $0,6 \mathrm{a}$ & $7,0 \mathrm{~b}$ & $5,2 \mathrm{~b}$ & $9,3 \mathrm{a}$ \\
\hline Tangelo 'Orlando' & $10,1 \mathrm{a}$ & $6,8 \mathrm{a}$ & $25,9 \mathrm{a}$ & $4,1 \mathrm{~b}$ & $13,3 \mathrm{ab}$ & $18,4 \mathrm{ab}$ & $69,6 \mathrm{a}$ & 148,3 a & $0,5 \mathrm{a}$ & $10,6 \mathrm{a}$ & $9,4 \mathrm{a}$ & $7,5 \mathrm{a}$ \\
\hline Tangerina 'Cleópatra' & $7,3 \mathrm{a}$ & $1,40 \mathrm{a}$ & $17,7 \mathrm{a}$ & $7,6 \mathrm{~b}$ & $15,2 \mathrm{ab}$ & $13,5 \mathrm{ab}$ & $61,5 \mathrm{a}$ & $124,2 \mathrm{a}$ & $0,5 \mathrm{a}$ & $10,6 \mathrm{a}$ & $7,1 \mathrm{ab}$ & $8,8 \mathrm{a}$ \\
\hline Média & 10,2 & 3,3 & 22,1 & 11,5 & 14,1 & 17,6 & 60,9 & 139,6 & 0,5 & 9 & 6,8 & 9,2 \\
\hline C.V. $(\%)$ & 42 & 203 & 26 & 72 & 60 & 40 & 28 & 25 & 34 & 14 & 22 & 30 \\
\hline
\end{tabular}

Médias seguidas da mesma letra na coluna não diferem, pelo teste de Tukey $(P<0,05)$ 
Acesso em: 20 set. 2006.

MATTOS JUNIOR, D.; DE NEGRI, J.; PIO, R.M.; POMPEU JUNIOR, J. Citros. Campinas: Instituto Agronômico e Fundag, 2005. 929 p.

OUKO, J.O.; ABUBAKER, A.S. Evaluation of citrus rootstocks in coast province of Kenya. Acta Horticulturae, Wageningen, v. 218, p. 43-50, 1988.

PARENTE, T.V.; WECHESLER, F.S.; BORGO, L.A.; REZENDE, L.P. Comportamento da tangerineira 'Ponkan'(Citrus reticulata Blanco) sobre 14 porta-enxertos do Distrito Federal. Revista Brasileira de Fruticultura, Cruz das Almas, v. 15, n. 1, p.35-41, 1993.

PERES, N.A., TIMMER, L.W. Evaluation of the Alter-Rater model for spray timing for control of Alternaria brown spot on Murcott tangor in Brazil. Crop Protection, Guildford v. 25, p. 454-460, 2005.

POMPEU JÚNIOR, J. Porta-enxertos. In: MATTOS JUNIOR, D.; DE NEGRI, J.D.; PIO, R.M.; POMPEU JUNIOR, J. (Org.). Citros. Campinas: Instituto Agronômico/FUNDAG, 2005. p. 63-104.

POMPEU JUNIOR, J.; BLUMER, S.; SALIBE, A.A. Tangerinas e tangelos como porta-enxertos para a laranja 'Hamlin'. Laranja, Cordeirópolis, v. 24, n. 2, p. 413-422, 2003.

REIS , R.F. Fatores relacionados à esporulação e período de suscetibilidade de folhas e frutos de tangerinas e híbridos a Alternaria alternata, agente causal da mancha marrom de alternaria. 2006. Tese (Doutorado em Agronomia, Produção Vegetal) - Faculdade de Ciências Agrárias e Veterinárias, Universidade Estadual Paulista, Jaboticabal, 2006.

SALIBE, A.A.; MOREIRA, C.S. Performance of Rangpur lime as rootstock for citrus in Brazil. In: INTERNATIONAL CITRUS CONGRESS, 1984, São Paulo. Proceeding... São Paulo: International Society of Citriculture, 1984. p. 29-33.

SMITH, M.W.; SHAW, R.G.; CHAPMAN, J.C.; OWEN-TURNER, J.; LEE, L.S.; McRAE, K.B.; JORGENSEN, K.R.; MUNGOMERY, W.V. Long-term performance of 'Ellendale' mandarin on seven commercial rootstocks in sub-tropical Australia. Scientia Horticulturae, Amsterdam, v. 102, p. 7589, 2004.

SOUZA, E.L.S.; PORTO, O.M.; RECK, S.R.; BRAUN, J. Comportamento do tangor 'Murcote' em 12 porta-enxertos no Rio Grande do Sul. Revista Brasileira de Fruticultura, Cruz das Almas, v. 14, n. 3, p. 105-112, 1992.

SPÓSITO, M.B.; CASTRO, P.R.C.; AGUSTÍ, M. Alternância de produção em citros. Laranja, Cordeirópolis, v. 19, n. 2, p. 293-304, 1998.

SPÓSITO, M.B., FEICHTENBERGER, E., PIO, R.M., CASTRO, J.L.; RENAUD, M.S.A. Ocorrência de mancha marrom de Alternaria em diferentes genótipos de citros nos estados de Minas Gerais, São Paulo e Rio Grande do Sul. Fitopatologia Brasileira, Brasília v. 28, p. 231, 2003.

SYVERTSEN, J.P.; GRAHAM, J.H. Hydraulic conductivity of roots, mineral nutrition, and leaf gas exchange of citrus rootstocks. Journal of the American Society for Horticultural Science, Geneva, v. 110, n. 6, p. 865-869, 1985. SYVERTSEN, J.P.; LLOYD, J.L. Cítrus. In. SHAFFER, B.;
ANDERSEN, P.C. Handbook of enviromental physiology of fruit crops. Boca Raton, Fla: CRC Press, 1994. chap. 4, p. 6599.

STENZEL, N.M.C.; NEVES, C.S.V.J.; GOMES, J.C.; MEDINA, C.C. Performance of 'Ponkan' mandarin on seven rootstocks in Southern Brazil. Hortscience, St. Joseph, v. 38, n. 2, p. 176178, 2003.

TIMMER, L.W., SOLEL, Z., GOTTWALD, T.R.; IBANEZ, A.M.; ZITKO, S.E. Environmental factors affecting production, release, and field populations of conidia of Alternaria alternata, the cause brown spot of citrus, Phytopathology, Lancaster, v. 88, n. 11, p. 1218-1223, 1998.

WUTSCHER, H. K. Rootstocks effects on fruit quality. In: FERGUSON, J.J.; WARDOWSKI, W. F. Factors affecting fruit quality. Lake Alfred: University of Florida, 1988. p. 2434.

WUTSCHER, H.K; SHULL, A.V. Performance of 13 citrus cultivars as rootstocks for grapefruit. Journal of the American Society for Horticultural Science, Geneva, v. 97, n. 6, p. 778-781, 1972.

WHEATON, T.A.; CASTLE, W.S.; TUCKER, D.P.H.; WHITNEY, J.D. Higher density for Florida citrus - concepts. Proceedings of the Florida State Horticultural Society, Tallahasse, n. 91, p. 17-33, 1978.

WHEATON, T.A. Alternate bearing of citrus in Florida. In: FUTCH, S.H.; KENDER W.J. (Ed.). Citrus flowering and fruiting short course. Lake Alfred: UFLA, IFAS, Citrus Research and Education Center, 1997. p. 87-92. 